\title{
Avaliação da Qualidade da Atenção à Saúde na Rede Básica
}

Paulo Roberto de Madureira*
Eduardo Mello De Capitani*
Gastão Wagner de Souza Campos*

A partir de uma análise de tendências quanto à organização dos serviços de saúde dentro da proposta de ações integradas de saúde, os autores se propõem a avaliar qualitativamente um serviço de saúde específico no município de PaulínialSão Paulo, que desde 1974 vem consolidando sua rede básica dentro de um sistema hierarquizado de atendimento com atençāo primária como porta de entrada, privilegiando a estrutura epidemiológica da população como base para o planejamento das ações de saúde. Os autores se propõem discutir alguns indicadores de qualidade de serviço de atenção ambulatorial na área de adultos, visando ultrapassar a visáo estreita de avaliação da produção de serviços até hoje em uso na rede pública. São analisados dados provenientes de 663 consultas médicas de adultos de primeiro atendimento referentes a diagnósticos, exames laboratoriais, esquemas terapêuticos e destino do paciente após a consulta. Valorizou-se na discussão as relaçôes $n$ : de consultas realizadas/ $n$ : de exames subsidiários solicitados; $n$. de medicamentos prescritos; $n$ : de consultas $n^{\circ}$. de consultas com prescrição; $n$ : de consultas $/ n$ : de retornos pedidos, indices estes relativizados pela qualidade dos diagnósticos realizados. Ressaltam que, apesar de-experimentais esses indices mostram-se relativamente adequados para a aferição preliminar da qualidade de um serviço ambulatorial a nível primário, superando a duvidosa eficácia de índices do tipo "resolutivia"ade", e apontam para a necessidade de novos estudos nesse sentido, visando a implementação da rede pública dentro do sistema unificado de saúde.

\section{INTRODUÇĀO}

A adoção dos pressupostos do Programa de Ações Integradas de Saúde como política oficial do Estado brasileiro tem imprimido alterações no modo de desenvolvimento da produção de serviços médico-sanitários. Entre outros aspectos interessa-nos ressaltar a ênfase que passou a ser dada ao atendimento ambulatorial, 
a se realizar através das unidades sanitárias públicas que compóem o que se convencionou chamar de rede básica ou de serviços de atenção primária à saúde.

A partir de 1983, o Ministério da Previdência e Assistência Social, através do INAMPS, vem repassando recursos financeiros aos govemos municipais e estaduais objetivando com isso reorientar o sentido que vinha tendo a expansão de cobertura da assistência médica, principalmente através de contratação de serviços hospitalares, intentando agora desenvolver o atendimento ambulatorial. Pretende-se dar à rede básica a função de operar como porta de entrada de um Sistema Integrado de Saúde (MPAS, 1983; MPAS/ CONASP, 1983).

Esta nova atribuição coloca um desafio para os Centros e Postos de Saúde que compõem a rede básica, o de alterar substancialmente seu perfil de atendimento e como decorrência, também redefinir uma nova composição de recursos. Tradicionalmente essas unidades organizaram-se a partir de uma concepção que lhes atribuía basicamente funçōes próprias ao campo da saúde pública desenvolvendo prioritariamente açōes de caráter coletivo. Assim sua estrutura capacitava-se a desenvolver programas de puericultura, pré-natal, controle de doenças endêmicas e outras atividades voltadas para a promoção e/ ou prevençãode quaủros mórbidos, aqui utilizando uma linguagem própria a esse entendimento, que recorta os serviços de saúde em dois campos: o da atenção indiviđual e o da saúde pública. A rede básica caberia essa segunda instância de atuação (CASTELLANOS, 1977; MOULAIN et al, 1985).

$O$ que significa funcionar como porta de entrada de um sistema de saúde? Segundo recomendaçōes da OMS, e em certa medida já incorporadas como parâmetros pelo INAMPS e por outras instituiçōes públicas, implica a capacidade destas unidades resolverem pelo menos $80 \%$ dos problemas de saúde da população de sua área programática. Adotando-se essa conceituação, há que se repensar a estrutura desses serviços tendo-se em conta năo mais conteúdos definidos "a priori" e de forma abstrata, mas considerando-se as "necessidades de saúde" dos usuários do sistema.

Existe toda uma controvérsia sobre a metodologia a ser empregada no diagnóstico de "necessidades de saúde", alguns autores privilegiando instrumentos técnicos que permitam identificar um determinado quadro epidemiológico através de exame de taxas de morbidade ou mortalidade, outros recomendando a combinação destas alternativas com um processo de ausculta da sociedade civil. De qualquer forma passou-se a entender que a rede básica deve organizar-se a partir destes diagnósticos de necessidades. A tecnologia ne- 
cessária ou adequada à A tenção Primária, segundo esta perspectiva, passaria a ser definida conforme a estrutura epidemiológica de cada região e, obviamente, em segunda instância, conforme a disponibilidade de recursos (PAIM, 1982).

A capacidade de resolver pelo menos $80 \%$ dos problemas de saúde só será alcançada em cada unidade, a partir da incorporação de uma determinada composição adequada de recursos, capaz de atingir aquela capacidade de resolução com eficácia e utilizando o mínimo de investimentos possíveis (SIAMENT, 1983). Obriga-as, além das tradicionais ações de saúde públi$\mathrm{ca}, \mathrm{a}$ incorporar a assistência médica individual à criança e ao adul to, dando conta das intercorrências clínicas que no modelo vigente no Brasil ainda caem principalmente nos Pronto Atendimentos localizados em hospitais contratados pela Previdência.

Assim, a vigir essa nova política, para operacionali zá-la há que se implan tar na rede básica n ovas rotinas, normas diagnósticas e terapêuticas, há que se redefinir a padronização de equipamentos médicos e de medicamentos à disposição da atenção primária à saúde.

Nas regiōes mais urbanizadas, onde ao lado das doenças infecciosas têm importância os danos à saúde decorrentes do modo de produção capitalista, como é o caso do município de Paulínia, onde se localiza - Centro de Saúde-Escola,objeto de estudo, e redefinição dos perfis das unidades ambulatoriais toma-se imperiosa. Nestas circunstâncias ampliam-se as necessidades de incorporação de tecnologias, particularmente nas áreas de apoio diagnóstico (laboratorial e radiológico), de reorganização do processo de trabalho e de redefinição dos padrōes anteriormente adotados no atendimento. Mesmo no caso das características da força de trabalho empregada na Atençāo Primária há que se reelaborar conceitos que passaram a desfrutar "status" de regras. Da utilização de médicos generalistas tende-se a trabalhar com" "gen eralistas especializados" em saúde da criança, da mulher e em clínica médica de adultos. Das auxiliares de saúde a incorporação de técnicas em enfermagem e enfermeiras. Da discussão sobre altemativas simplificadas para saneamento (fossas, cloração de poços, entre outras) intenta-se passar para a discussão do direito à saúde e em decorrência, do saneamento básico; às aulas de higiene pessoal sucedem abordagens decodificadas do processo de saúde e doença, do incentivo ao autocuidado discute-se, em várias experiências, os diferentes usos sociais do corpo e as políticas de promoção e recuperação da saúde.

Enfim, tenta-se, em várias experiências que se propōem reformar a Atençāo Primária à Saúde, fazer a negação/superação do tradicional papel das unidades 
sanitárias, através da incorporação da assistência médica individual e de todos os pressupostos tecnológicos que atualmente a definem. Ao mesmo tempo tenta-se a negaçāo/superaçāo dos limites da assistência médica individual, utilizando elementos da crítica a essa prática desenvolvida pela medicina social, discutindo-se a "medicalização" e a ênfase curativa e centrada no indivíduo, características da "medicina científica" (DONNANGELO, 1976).

Nessa perspectiva, entendemos ser de crucial importância a tarefa de definir novos parâmetros de avaliação de qualidade desses serviços de atenção primária, no intuito de se implementar a atual política de saúde.

Os parâmetros oficialmente adotados para avaliação do desempenho de serviços de saúde têm se atido, via de regra, a quantificar a produção das unidades, a indicadores de produtividade à análise das capacidades de utilização de equipamentos e instalações. Quando muito, propóem valiaçóes da cobertura populacional e de concentração de atividades (MS, 1985; GRÉ MY, 1984; DENISTON et al, 1969).

Entendemos que não há separação intransponível entre os indicadores quantitativos e qualitativos, ao contrário, na perspectiva de que é o acúmulo de avaliações quantitativas que permite aproximações progressivas de uma "idéia de qualidade na prestação de serviços", é que sugerimos a incorporação de novos parâmetros na avaliação das unidades de saúde. Orientamonos na construção e adoção dessas altemativas pela preocupação em avaliar criticamente o desempenho dos serviços de saúde, verificando em que medida superamos a medicalização própria da prática médica "científica", e verificando até que ponto nosso Centro de Saúde superou a organizaçẫo tradicionalmente preconizada pelos Programas de Saúde Pública.

Nesse sentido, procuramos responder algumas questões pertinentes ao funcionamento do CSEP: que tipo de paciente e que problemas de saúde atendemos? Qual a composição tecnológica que utilizamos para desenvolver nossas atividades? Como nos utilizamos dos serviços de laboratório e radiodiagnóstico? $\mathrm{Na}$ abordagem geral do paciente, que peso tem a terapêutica medicamentosa? Que apoio hospitalar e especializado demandamos?

\section{MATERIAL E MÉTODOS}

O Centro de Saúde Escola de Paulínia funciona desde 1974 sendo que há cerca de 5 anos $80 \%$ da população de sua área programática o utiliza quando demanda asssitência ambulatorial, constituindo-se como primeira linha de atendimento do sistema público 
que se logrou organizar na região. Paulínia é um município do Estado de São Paulo, com cerca de $35 \mathrm{mil}$ habitantes com características industriais bem marcadas pela presença de importante pólo petroquímico. Sua rede básica atual é composta de um Centro de Saúde com três Postos de Saúde periféricos e um hospital secundário que atua a nível de região. Sua atenção terciária fica por conta do Hospital Universitário da Unicamp. A atenção médica a nível privado é bastante reduzida, limitando-se a alguns consultórios e um hospital pequeno que mantém alguns convênios com pequenas empresas da regiāo.

Os dados do presente trabalho foram coletados a partir de 663 consultas médicas realizadas na área de clínica de adultos do CSEP. Foram obtidas informações de ficha preenchida pelo médico ou estagiário médico durante 0 ato da consulta que contemplavam, além de dados de identificação demográfica, diagnósticos realizados; exames subsidiários solicitados; esquemas terapêuticos ministrados e destino do paciente após a consulta.

Os indicadores a serem trabalhados tentavam, de certa forma, superar impasse qDi o termo "resolutividade" vem trazendo às avaliações de serviço, desde que não qualifica a demanda aceita e "resolvida" pelo serviço e aquela "encaminhada" e não resolvida, ou seja, podemos ter um serviço que só encaminha $5 \%$ de sua demanda (sendo considerado um ótimo serviço por isso) porque nāo tem condições e recursos para diagnosticar a necessidade real de encaminhamentos. Por outro lado, um serviço que encaminha $20 \%$ de sua demanda pode ser um bom serviço, à luz de seus recursos, desde que está diagnosticando essa necessidade de encaminhamentos.

Os indicadores propostos relacionam o número de consultas realizadas com o número de exames subsidiários solicitados; com o número de consultas que necessitaram de exames subsidiários; com o número de medicamentos prescritos por consulta; com o número de consultas sem prescrição de medicamentos e com o número de retornos pedidos para seguimento. Esses indicadores devem, no entanto, serem relativizados pela qualidade dos diagnósticos realizados para funcionarem como parâmetros de qualidade.

\section{RESULTADOS E DISCUSSÃO}

As Tabelas 1,2 e 3 mostram dados que melhor caracterizam a demanda incluída no estudo. Convém ressaltar a quase equivalência entre os sexos, caracterizando demanda que foge ao perfil tradicional da busca de atendimento nas unidades sanitárias que é de predomínio de mulheres em idade fértil. A concentração 
da demanda masculina na faixa etária produtiva da, vida (Tabela 3 ) reforça essa tendência de mudança de perfil da demanda tradicionalmente atendida.

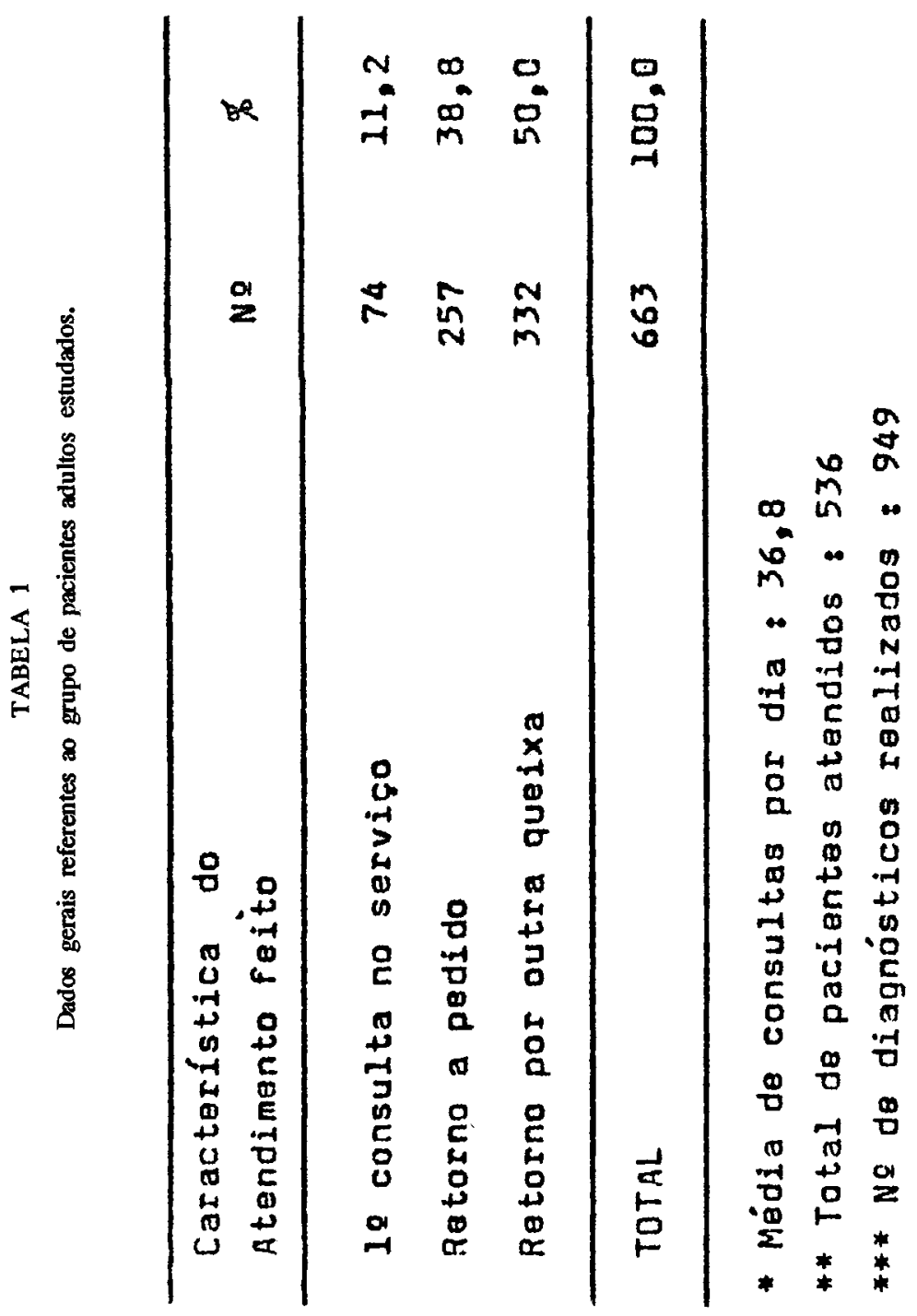




\section{TABELA 2}

Distribuiçäo dos pacientes atendidos segundo o sexo.

\begin{tabular}{lll}
\hline Sexo & Ne & \% \\
\hline Masculino & 241 & 44,9 \\
Feminino & 295 & 55,1 \\
\hline TOTAL & 536 & 100,0 \\
\hline
\end{tabular}

TABELA 3

Distribuição dos pacientes atendidos do sexo masculino por faixa etária.

\begin{tabular}{lcc}
\hline Faixa Etária & No & $\%$ \\
\hline $10-20$ & 35 & 14,5 \\
$20-30$ & 59 & 24,4 \\
$30-40$ & 63 & 26,1 \\
$40-50$ & 32 & 13,2 \\
$50-60$ & 23 & 9,6 \\
$60-70$ & 29 & 12,2 \\
\hline TOTAL & 241 & 100,0 \\
\hline
\end{tabular}

Cadernos de Saúde Pública, RJ, 5 (1): 45-59, jan/mar, 1989 
Na Tabela 4 vemos dispostos os dados referentes à solicitação ou não de exames subsidiários. Notamos que em $54 \%$ das consultas não foi solicitado qualquer tipo de exame, o que caracteriza um baixo índice de solicitaçăo que se compararmos ao preconizado pela Portaria 3046/82 (MPAS/CONASP, 1983) que é de $70 \%$. Aqui a primeira necessidade de relativizar-se esses índices com os dados referentes a diagnósticos, pois esse baixo índice encontrado é explicado, de um lado, pela alita porcentagem de patologias crônicas deste grupo de pacientes estudados, quando a avaliação diagnóstica laboratorial já foi realizada. Outro fator explicativo desse índice é o estímulo dado pelo serviço ao seguimento clínico dos doentes, evitando-se a solicitaçẫo de exames que possam ser prescindidos por uma nova consulta. Entendemos que o uso de exames subsidiários na prática clínica está diretamente ligado ao acesso que o serviço tenha à realização dos mesmos $e$, inversamente relacionado ao nível de qualidade do profissional envolvido com essa prática.

TABELA 4

Distribuiçāo das consultas estudadas segundo o número de exames laboratoriais solicitados.

$\begin{array}{ll}\text { Número de exames } & \text { Número de } \\ \text { Solicitados } & \text { Consultas }\end{array}$

\begin{tabular}{crr}
\hline Nenhum & 341 & 54,0 \\
1 & 178 & 28,0 \\
2 & 74 & 12,0 \\
3 & 22 & 3,5 \\
$>3$ & 16 & 2,5 \\
\hline TOTAL & 663 & 100,0 \\
\hline
\end{tabular}


A Tabela 5 completa a discussão acima trazendo dados referentes à utilização de exames complementares não laboratoriais. Verificamos que o tipo de exame solicitado se sobrepōe à real necessidade frente aos diagnósticos realizados (ver Tabela 9). Ressaltamos que exames do tipo Urogafia Excretora, Ecografia abdominal, Endoscopia digestiva e Biópsia de Pele, tradicionalmente a cargo da atenção secundária e terciária mostram-se de extremo valor na resolução da demanda, além de servirem como estímulo de reciclagem dos profissionais da rede que vêem seus intentos diagnósticos contemplados tecnicamente.

TABELA 5

Distribuição das consultas realizadas segundo o tipo de procedimento diagnóstico complementar solicitado.

Tipo de Procedimento No $\quad 8$

Nenhum Procedimento

ECG

RX de Tórax

PPO

Endoscopia de TGI

Ecografia Abdominal

Urografia Excretora

RX de Coluna Vertebral

Abrougrafia

$R \times$ de 03908

Biópsia da Pele
565

85,2

30

4,5

29

4,4

18

2,7

5

0,8

4

0,6

3

0,5

3

0,5

2

0,3

20,3

20,3

\section{TOTAL}

663100,0 
$\mathrm{Na}$ Tabela 6, quando analisamos o índice de prescrição de medicamentos por consulta médica, vemos que $25,0 \%$ dos pacientes não receberam nenhuma prescrição. Sabendo que a prescrição de medicamentos é o código de ligação entre paciente e serviço de saúde, servindo muitas vezes para substituir a relação médico/paciente devido ao alto grau de medicalização em vigor na prática de saúde, entendemos que a valorização desse índice como indicador de certa transformação dessa prática e de qualidade do serviço desde que reflete a utilização de orientações sobre a patologia, orientaçōes higieno-dietéticas, fisioterápicas etc que substituem a prescrição de medicamentos, melhorando a vinculação do paciente ao serviço.

\section{TABELA 6}

Distribuição das consultas realizadas segundo o número de medicamentos prescritos.

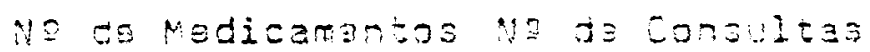

\begin{tabular}{|c|c|c|}
\hline id 3 rnum & 155 & 25,5 \\
\hline 1 & 322 & $4, \overline{3}$ \\
\hline 2 & 115 & $\pm \hat{i}$ \\
\hline 3 & 41 & 6,3 \\
\hline$>3$ & $2=$ & 3,5 \\
\hline TUTA: & 663 & 290 \\
\hline
\end{tabular}

Quanto aos encaminhamentos a especialidades, temos na Tabela 7 que do total de consultas, $4,2 \%$ necessitou de encaminhamento, com predomínio para Psiquiatria, Oftalmologia e Ortopedia, dados que estão de acordo com outros serviços que realmente funcionam como porta de entrada para o sistema (SIAMENT, 1983). A porcentagem baixa de encaminhamentos às especialidades talvez reflita o bom acesso a exames subsidiários em geral do serviço aliado à formação clínica do pessoal que presta o serviço. 
Distribuição dos pacientes encaminhados segundo a especialidade.

\begin{tabular}{|c|c|c|}
\hline Espacialidade & No & $\%$ \\
\hline Psiquiatria & 8 & $2 \mathrm{E}, 6$ \\
\hline oftalmologia & 4 & 14,3 \\
\hline Neurologia & 3 & 11,0 \\
\hline Ortopedia & 2 & 7,2 \\
\hline Dermatologia & 2 & 7.2 \\
\hline Endocrinologia & 1 & 3,6 \\
\hline Gastrocirurgia & 1 & 3.6 \\
\hline Nefrologia & 1 & 3,6 \\
\hline Urologia & 1 & 3,6 \\
\hline Cardiologia & 1 & 3,6 \\
\hline otorrinilaringologia & 1 & 3,6 \\
\hline Medicina do Trabalho & 1 & 3,6 \\
\hline Cirurgla plástica & 1 & 3,6 \\
\hline$B M F$ & 1 & $3: 6$ \\
\hline TOTAL & 28 & 100.3 \\
\hline
\end{tabular}

A Tabela 8 traz uma listagem de diagnósticos realizados com suas respectivas percentagens. Chama a atenção o padráo de morbidade mista entre patologias degenerativas e infecto-contagiosas, de acordo com o padráo epidemiológico de nosso meio.

Cadernos de Saúde Pública, RJ, 5 (1): 45-59, jan/mar, 1989 
TABELA 8

Distribuiçăo percentual dos diagnósticos realizados.

\begin{tabular}{|c|c|c|}
\hline Diagnósticos & NQ & 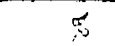 \\
\hline $4 i p 3 r \operatorname{tansä0}$ nrtarial & 122 & 13,9 \\
\hline risponsia & 80 & 8,5 \\
\hline Sireiadade & 69 & 7,3 \\
\hline 2o:natopatias & 53 & 5,6 \\
\hline Far 35 itoses intestinais & 49 & $5, ?$ \\
\hline $\operatorname{Lon} 2 \approx 10 \mathrm{i} a 3$ & 45 & 4,7 \\
\hline Afecooss otortinolaringológicas & 41 & 4,3 \\
\hline $\operatorname{linj}$ & 40 & $4, ?$ \\
\hline Infozçõos do Trato Urinário & 40 & 4,2 \\
\hline Cardiopatias & 3 月 & 4,0 \\
\hline Freumopatias (axcato TBC) & 30 & 3,1 \\
\hline Oiabete Mallitus & 26 & 2,7 \\
\hline Cofalélas & 26 & 2,7 \\
\hline Jindrome anêmica & 24 & 2,5 \\
\hline Doencas Nefrólurológlcas & 19 & 2,0 \\
\hline Obesldade & 29 & 2,0 \\
\hline Leucorrálas & 18 & 1,9 \\
\hline TBC & 19 & 1,9 \\
\hline Afoç̧öes Ortopódicas & 17 & 1,8 \\
\hline Afrocõos Reumatolózicas & 17 & 1,8 \\
\hline Dorngas Sexualmante Transmissív. & - 12 & 1,3 \\
\hline Colopatias funcionats & 13 & 1.4 \\
\hline Afeccões oftalmológicas & 11 & 1,2 \\
\hline Cas;roanterocolitas & 11 & 1,2 \\
\hline Loarrast Montas & 11 & 1,2 \\
\hline Esquistossomoso & 20 & 1,0 \\
\hline Eplepsia & 9 & 0,9 \\
\hline Molóstia de Hansen & 7 & 0.7 \\
\hline Afeccoos Naurológicas & 7 & 0,7 \\
\hline Arações Vascularas & 6 & 0,6 \\
\hline Dutzos & 61 & 6,4 \\
\hline TOTAL & 949 & 100,0 \\
\hline
\end{tabular}




\section{CONCLUSÕES}

Uma questão difícil de ser superada, quando se intenta realizar análises qualitativas de serviços de saúde no Brasil, decorre da ausência de regionalização e/ou hierarquizaçāo da assistência médico-sanitária, e conseqüentemente inexistência de definições quanto à cobertura populacional de cada unidade. Neste esquema os pacientes tendem a utilizar-se de vários serviços durante determinada intercorrência clínica, acumulando-se assim, efeitos decorrentes da atuação de diversas abordagens. Isto sem mencionar a dificuldade metodológica de se medir o impacto de serviços de saúde provenientes dos próprios determinantes do processo saúde/doença que transcendem aqueles com origem exclusivamente na atenção médica.

O município de Paulínia, área programática do CSEP, não foge a essa regra. Contudo, alguns dados encontrados evidenciam que, não obstante o atual estágio de desenvolvimento da política nacional de saúde, a referida unidade já funciona como porta de entrada, prestando primeiro atendimento a pelo menos $80 \%$ da população prevista, conseguindo fugir, em certa medida, aos padrões tradicionais de funcionamento da rede básica. A distribuição da demanda do CSEP, por faixa etária e por sexo, na faixa dos adultos, não segue a lógica dos programas tradicionais usualmente recomendados e adotados na rede básica, em que predominam o atendimento "preventivo" de pré-natal e controle de doenças infecto-contagiosas endêmicas. No caso do CSEP, atende-se tanto o adulto jovem em idade produtiva, quanto o idoso; não só as mulheres dessa faixa etária mas também os pacientes masculinos, o que confirma o papel da porta de entrada da unidade.

Os diagnósticos realizados mais prevalentes evidenciam que não predominam, entre os adultos atendidos, processos mórbidos considerados objeto de intervençấo específica do campo da saúde pública, e sim aqueles mais freqüentemente encontrados entre populaçōes que vivem em regiōes industrializadas e com altas taxas de urbanização, como as doenças crônicodegenerativas que no caso brasileiro demandavam assistência predominante na área hospitalar.

Usualmente a "capacidade resolutiva" é apontada como o principal indicador de eficácia, de resolver problemas de saúde, de evitar danos. Capacidade resolutiva ou "resolutividade" é geralmente definida como a porcentagem de casos que tiveram atendimento terminal no serviço em estudo, ou seja, a porcentagem de pacientes que não foram encaminhados para atendimento especializado. $\mathrm{O}$ que se tentou com o presente estudo foi justamente "qualificar" essa capacidade resolutiva enriquecendo-a com indicadores extraídos do 
próprio atendimento, do próprio ato da consulta. Entendemos, no entanto, tratar-se de análise dentro de um contexto que pode relativizar seus resultados, sendo apenas um início de proposta que esperamos seja adendado com dados de outros serviços para que cheguemos à definição de critérios de avaliação de qualidade de serviço mais reproduzíveis.

The authors going through an analyses of the trends in current policy of integration of health services in the public field in Brazil, propose a qualitative assessment of these services as an imperative need at this point of implementation of that policy. They focused the Health Center of Paulinia in the state of São Paulo that since 1974 has been running an integrated system where the primary atention is the first door the patient hits searching for health care. Trying to work with qualitative parameters of medical attention they analysed 663 adult consultations, examining their diagnostic pattern, number and type of medicine prescribed, number of complementary laboratory examens and the further destination of the patient. Some indices as number of consultations done/number of complementary laboratoty examens; $\mathrm{NC} /$ number of medicine prescribed $\mathrm{NC} / \mathrm{NC}$ with medicine prescriptions and NC/ number of asks for return in the service for a follow-up, were stablished. All these indices were related with the pattern of diagnostic done. Despite the simplicity of these experimental indices the authors believe they are good enough to detect services in a good path of quality from others whose goal is simply production of consultation as an answer to pressures of a social demand. They also believe further studies must be developed in this field trying to improve the health care in the Brazilian net of public services.

\section{REFERÊNCIAS BIBLIOGRÁFICAS}

CASTELLANOS, J. Situaciones de cobertura, níveles de atención y atención primária. Bol. Of. Sanit. Panam. 82: 478-92, 1977.

DENISTON, O. L. et al. Evaluación del rendimento de programas de salud. Bol. Of. Sanit. Panam.: 389-398 Nov. 1969.

DONNANGFLO, M. C. F. \& PEREIRA, L. Saúde e Sociedade.São Paulo Editora Livraria Duas Cidades, 1976.

GRE MY, F. Evaluation of medical action and action in public health. Effec. Health. Cate, 1: , 1983.

LLOVET, I. J. Servicios de salud y sectores populares: los años del proceso. Buenos Aires, Edición Cedes, 1984. 
MINISTÉRIO DA PREVIDÊNCIA E ASSISTÊNCIA SOCIAL/ CONASP - Reorientação da assistência à saúde no âmbito da Previdência Social. Documento Oficial, 1983.

MINISTÉRIO DA PREVIDÊNCIA SOCIAL/ MINISTÉRIO DA SAÚDE/SECRETARIAS ESTADUAIS DE SAÚDE - Açōes Integradas de Saúde, Documento Oficial, 1985.

MOULAIN, M. et al. Orientação para organização de Centros de Saíde: o planejamento local. Brasília, Centro de Documentação do MS, 1985.

PAIM, J. As ambigüidades da noção de necessidades de saúde. Salvador, FCM da UFB, 1982.

SIAMENT, S. J. Estudio de la capacidade resolutiva de la demanda de morbidad a nivel primario. (Área urbana). Cuad. Med. Soc. 24: $170-175,1983$. 